Published in final edited form as:

J Am Geriatr Soc. 2012 May ; 60(5): 922-926. doi:10.1111/j.1532-5415.2012.03927.x.

\title{
Racial Differences in Gait Velocity in an Urban Elderly Cohort
}

\author{
Irene Blanco, MD, MS ${ }^{1}$, Joe Verghese, MB BS ${ }^{2,4}$, Richard B. Lipton, MD ${ }^{2,3,4}$, Chaim \\ Putterman, $\mathbf{M D}^{1}$, and Carol A. Derby, $\mathbf{P h D}^{2,3,4}$ \\ ${ }^{1}$ Division of Rheumatology, Albert Einstein College of Medicine, Bronx, NY \\ ${ }^{2}$ Department of Neurology, Albert Einstein College of Medicine, Bronx, NY \\ ${ }^{3}$ Department of Epidemiology and Population Health, Albert Einstein College of Medicine, Bronx, \\ NY \\ ${ }^{4}$ Einstein Aging Study, Albert Einstein College of Medicine, Bronx, NY
}

\section{Abstract \\ Background/ Objectives-Gait Velocity (GV) is predictive of hospitalizations and mortality in the elderly. In the US, elderly African Americans have higher rates of physical disability compared to Caucasians. Few studies have investigated if there are racial differences in GV in the elderly.}

Design-We performed a cross sectional analysis to investigate racial differences in GV.

Setting/Participants-Participants were part of the Einstein Aging Study, a longitudinal study of community-residing elderly in the Bronx, NY. They were recruited using Medicare and voter registration records. Records of 213 participants were analyzed.

\begin{abstract}
Measurements-Demographics, medical history, the Geriatric Depression Scale, the Blessed Information Memory Concentration Test, and the Total Pain Index (TPI) were collected. GV was measured using the GAITrite ${ }^{\circledR}$ mat.
\end{abstract}

\begin{abstract}
Results-We included 157 Caucasians and 56 African Americans. Caucasians were older (median 79.9y v 75.5y, $\mathrm{p}<0.01$ ), more educated (median $14 \mathrm{y} \vee 12 \mathrm{y}, \mathrm{p}<0.01$ ) and had lower BMIs (mean 26.9 \pm 4.3 v $28.9 \pm 6.4, \mathrm{p}=0.03$ ). African Americans had higher proportions of female participants $(80.4 \%$ v $59.9 \%, \mathrm{p}<0.01)$ and diabetes $(28.6 \%$ v $13.4 \%, \mathrm{p}=0.01)$. Neither group had significantly higher pain levels. African Americans had a significantly slower GV (mean $90.2 \pm 17.9 \mathrm{v} 99.1 \pm 20.1 \mathrm{~cm} / \mathrm{sec}, \mathrm{p}<0.01$ ). This difference persists despite adjusting for multiple covariates. A $7.79 \mathrm{~cm} / \mathrm{sec}$ slower gait speed in African Americans versus Caucasians was not explained by differences in common factors known to influence gait.
\end{abstract}

Conclusion-Differences in GV persist between African Americans and Caucasians despite adjusting for many confounders. Increases of just $10 \mathrm{~cm} / \mathrm{sec}$ are associated with reduced mortality. Further studies are needed to evaluate if there are modifiable risk factors that potentially explain this difference and if an intervention could reduce the discrepancy between the groups.

\footnotetext{
Corresponding Author: Irene Blanco, MD, MS, Division of Rheumatology, Albert Einstein College of Medicine, Forchheimer 701, 1300 Morris Park Ave., Bronx, NY 10461, irene.blanco01@gmail.com, Telephone: 718-430-2894, Fax: 718-430-8789. Alternate Corresponding Author: Carol Derby, PhD, carol.derby@einstein.yu.edu.

Conflict of Interest

Author Contributions: All authors significantly contributed to study concept and design, acquisition of data, analysis and interpretation of data, and preparation of manuscript.
} 


\section{Keywords}

gait velocity; health disparities; elderly; physical function

\section{INTRODUCTION}

Despite multiple public health efforts, racial disparities in health outcomes persist. ${ }^{1}$ African

Americans are more likely to have higher rates of physical disability as compared to Caucasians. ${ }^{2,3}$ In a study of high functioning elderly women, African Americans who were preclinically disabled were more likely to be disabled 18 months later as compared to their Caucasian counterparts. ${ }^{4}$ These differences may be explained by socioeconomic (SES) factors as several studies have suggested. However others have shown that differences persist after adjustment for SES. ${ }^{5-8}$

Gait velocity, a functional measure that requires no special equipment and can be performed quickly in the clinical setting, is a validated measure of physical function. Decreases are strongly predictive of incident disability and nursing home admissions. ${ }^{9-11}$ Given its ease of use and predictive capability, gait velocity is a recommended screening tool for physical decline in the elderly. ${ }^{12}$ Despite observed racial differences in physical function, few studies have addressed whether gait velocity, an important predictor of disability differs by race. Therefore, we performed a cross-sectional study in the community-based, multi-ethnic cohort of the Einstein Aging Study to examine whether there are differences in physical function as measured by gait velocity among elderly African Americans and Caucasians.

\section{METHODS}

\section{Population}

Analyses are based on data from participants in the Einstein Aging Study (EAS) cohort who completed a baseline clinic evaluation between 2004 and 2009. The EAS is a longitudinal study of community residing elderly in the Bronx, NY which began in $1993 .{ }^{13,14}$ From 1993-2004, Medicare beneficiary lists from the Center for Medicare Services were used to generate systematic sampling frames for the selection of potential participants. Due to changes in access to these lists, beginning in 2004, participants were identified using voter registration lists for Bronx County, NY. English-speaking persons $\geq 70$ years were contacted first by letter and then by telephone. Individuals wishing to participate gave verbal consent and completed a brief telephone screening which included a medical history and cognitive screening tests. Participants who met eligibility criteria were invited for further evaluation at our clinical research center. Excluded from the EAS were those who were institutionalized, did not speak English, or who were unable to ambulate. Also excluded were those with sufficient audiovisual disturbance interfering with neuropsychological testing. Written informed consents were obtained during clinic visits using a protocol approved by the Institutional Committee on Clinical Investigations at Albert Einstein College of Medicine, Bronx, NY.

Clinical Assessments and Surveys-Data were collected by a computer assisted telephone interview and during in-person clinic visits using standardized protocols. ${ }^{13}$ Demographic information included: age, self-described race according to categories from the 2000 US census, and years of education. Body mass index was calculated using the measured height and weight of each subject. Past medical history was obtained in the form of, "Has a doctor ever told you that you have or have had any of the following conditions?" to which the participant answered either yes or no. The conditions included but were not limited to diabetes, heart attack, stroke, chronic obstructive pulmonary disease (COPD), 
osteoarthritis, hip replacement or pinning, or any of the following fractures: hip, femur or pelvis fracture.

Depression and cognition were assessed using the Geriatric Depression Scale (GDS) and the Blessed Information-Memory Concentration test (BIMC), respectively. ${ }^{15,16}$ The GDS was dichotomized at six with those scoring $\searrow 6$ classified as likely being depressed. ${ }^{17}$ The BIMC was dichotomized at a score of eight, with a score $\geq 8$ considered indicative of cognitive impairment. ${ }^{18}$

Pain was assessed using the Total Pain Index (TPI) which was administered during the telephone interview. The TPI assesses pain location, severity, duration and frequency over the 3 months prior to the interview in 8 different areas: head, face, neck and shoulders, back, arms and hands, legs and feet, chest, abdomen and pelvis. Pain for each area was assessed both as a 5-point Likert scale ranging from "none of the time" to "all of the time" and quantitatively as a numeric score. To get the summative TPI score, scores were summed across the body areas. ${ }^{13}$ For the purposes of this study, the TPI score was used as a continuous variable, and scores from specific body areas were dichotomized at "none of the time" to "slight bit of the time" and "some of the time" to "all of the time."

Gait Velocity Measurements-Quantitative gait measurements were conducted by research assistants using the GAITRite ${ }^{\circledR}$ mat, a 12-foot computerized gait mat embedded with pressure sensors. (CIRsystems, Havertown, PA). Participants were asked to walk on the mat at "their normal walking speed" down a well-lit hallway. Start and stop points were marked on the floor and 3' areas were included for acceleration and deceleration. Gait velocity was determined by dividing the distance traveled over 3 trials by the ambulation time. Our group and others have demonstrated reliable and reproducible gait assessments using this tool. 19,20

\section{Statistical Analysis}

All analyses were performed using STATA, 10.1 (STATA-Corp LP, College Station, TX.) Bivariate analyses were performed to compare characteristics for African Americans and Caucasians and to determine which variables were associated with gait velocity. Student's ttest was used to analyze the means for continuous variables. For categorical variables, the Chi-square test was performed. When data did not meet test assumptions, the MannWhitney U test was performed and the Fisher's exact test p-value was reported. Correlations between two continuous variables were evaluated using Pearson's correlation coefficient. The results of these analyses are shown in Tables 1 and 2.

To investigate racial differences in gait velocity while adjusting for covariates, five nested linear regression models were fit with gait velocity as the outcome variable. We included all variables shown to have at least a modest association with gait velocity ( $\mathrm{p}$-values of $<0.15$ on bivariate analyses). We also included variables that were deemed to be clinically significant regardless of their statistical significance because of their possible confounding relationship to gait, such as overall pain, back and lower extremity pain, hip replacement/ pinning and history of osteoarthritis. Model 1 is an unadjusted model with race as the sole independent variable. Model 2 includes race and was adjusted for age, gender and BMI. Model 3 includes the terms in Model 2 plus education as a proxy for SES. Model 4 was further adjusted for the following co-morbidities: depression, osteoarthritis, diabetes, stroke, hip replacement or pinning, or hip/pelvis or femur fracture. Finally, Model 5 included the pain variables: TPI, back pain, lower extremity pain, each tested separately. Variables were tested separately and in groups. All of the above linear regression models were tested for assumptions of normality, and collinearity. The results of this analysis can be found in Table 3. 


\section{RESULTS}

A total of 617 EAS participants underwent a baseline clinic visit between September, 2004 and November, 2009. Seventy-eight participants were excluded because they used an assistive device when ambulating. 326 participants were excluded due to missing covariate data which would preclude them from being part of the regression analyses. Data from 213 EAS participants were included in the final analysis. Overall, there was no statistically significant difference between those excluded and included in terms of age, gender, race, BMI, years of education and mean gait velocity and the following comorbidities: depression, dementia, heart attack, COPD, diabetes, stroke, hip replacement or pinning, or a history of hip/femur/pelvis fracture. There was no difference in median TPI scores between those included and excluded. Those included did have higher levels of osteoarthritis (76.0\% v $56.7 \%, \mathrm{p}<0.01)$, as well as higher levels of back pain $(33.3 \% \mathrm{v} 16.4 \%, \mathrm{p}<0.01)$, and lower extremity pain $(36.6 \% \mathrm{v} 16.1 \%, \mathrm{p}<0.01)$.

Descriptive characteristics of the study population by race are listed in Table 1 . The Caucasian group was significantly older and had a significantly higher percentage of male participants. Caucasians also had significantly lower mean BMI and more median years of education than did the African American group. African Americans had a significantly higher percentage of cognitive impairment as measured by the BIMC and a higher percentage of diabetes. There were no statistically significant differences between the two groups with regards to the other listed co-morbidities. (Table 1)

Overall the mean gait velocity was $96.7 \pm 19.9 \mathrm{~cm} / \mathrm{sec}$ for the entire cohort; $54.5 \%$ had a speed of $<100 \mathrm{~cm} / \mathrm{sec}$. There was a statistically significant difference between Caucasians and African Americans in terms of gait velocity. African Americans had an $8.87 \mathrm{~cm} / \mathrm{sec}$ slower mean gait velocity when compared to Caucasians $(\mathrm{p}<0.01)$. Bivariate analyses showed that for both African Americans and Caucasians, gait velocity was modestly inversely correlated with age (rho=-0.19, $\mathrm{p}<0.01)$, BMI (rho= $=0.26, \mathrm{p}<0.01)$ and cumulative number of the participant's comorbidities (rho $=-0.15, \mathrm{p}=0.03$ ). However gait velocity was not correlated with years of education or the TPI as a continuous variable. Gait velocity was significantly decreased in those with a history of depression, diabetes, and lower extremity pain. Further results can be found in Table 2.

We performed five nested linear regression analyses to evaluate the difference in gait velocity, between African Americans and Caucasians (Table 3). Despite adjusting for age, gender, BMI and education, gait velocity remained significantly associated with race. Race remained a significant predictor after additional adjustment for comorbidities and for pain indices. The effect of race was only slightly attenuated (12\%) when adjusting for all covariates in Model 5. Overall, gait velocity among African Americans was $7.79 \mathrm{~cm} / \mathrm{sec}$ slower than that for Caucasians when adjusting for multiple covariates that could confound its association with race. $(\mathrm{p}=0.011)$

\section{DISCUSSION}

In this study, we performed a cross-sectional analysis to evaluate differences in gait velocity between African Americans and Caucasians participating in the Einstein Aging Study. We found that gait velocity was approximately $8 \mathrm{~cm} / \mathrm{sec}$ slower among African Americans than Caucasians. This difference persists despite adjustment for multiple potential confounders and covariates shown to have an association with gait velocity on bivariate analyses.

Even in highly functional patients, a gait velocity of less than $100 \mathrm{~cm} / \mathrm{sec}$ is predictive of increased hospitalization rates. As gait velocity decreases, patients are at a higher risk for the need of a caregiver, falls and death. ${ }^{12,21}$ Small improvements in velocity, as little as 4-16 
$\mathrm{cm} / \mathrm{sec}$, can lead to meaningful improvements in physical function; where increases in gait velocity of $10 \mathrm{~cm} / \mathrm{sec}$ are associated with decreased mortality in those that are both independent and dependant in their activities of daily living. ${ }^{22,23}$

Overall, $54.5 \%$ of our entire study population had a gait velocity less than $100 \mathrm{~cm} / \mathrm{sec}$, and the mean gait velocity among African Americans was at this level. Given our group's finding that there is a $1.48 \mathrm{~cm} / \mathrm{sec} / \mathrm{year}$ overall decline in gait velocity in the EAS as a participant ages, African Americans who have a lower gait velocity at baseline may reach incident disability earlier than Caucasians, even with similar yearly rates of decline. ${ }^{24}$ Gait velocity may then be a potential target for intervention especially in African Americans, who have higher rates of disability.

It is difficult to know how exactly to target gait velocity given we are uncertain of modifiable predictors of this functional measure as stratified by race. Nevertheless, one could speculate that there is an inherent difference in gait and gait velocity in African Americans as compared to Caucasians. In a study of older adults with OA, despite the fact that African Americans and Caucasians had similar levels of physical function and mild OA on radiographs, when tested using a 6 minute walk test, African Americans fared worse. ${ }^{25}$ Another study evaluating gait mechanics in participants with knee OA showed that African Americans had worse gait parameters despite the same degree of OA on radiography even after adjusting for pain, disability, BMI, and OA severity. ${ }^{26}$

Because of the possible racial differences seen in OA, we did adjust our analyses for a history of OA. We found that when adjusting for other comorbidities that a history of OA was actually associated with increased gait velocity in both groups. This unexpected finding may be due to the fact that our self-reported variable of a past medical history of OA likely includes both upper and lower extremity disease. We corrected for this by including the presence of lower extremity pain as measured by the TPI in our analyses especially given that those with lower extremity pain had a statistically significant decrease in gait velocity as noted in table 2 . Nevertheless, adjustment for both OA and lower extremity pain did not explain the observed differences in gait velocity among African Americans and Caucasians.

Several studies have reported increased rates of pain in African Americans and this may explain differences in gait velocity. ${ }^{27} \mathrm{We}$ also observed that African Americans had a nonsignificant trend toward higher overall TPI scores when compared to Caucasians. There were however, no differences noted between the groups with regards to the individual components of the TPI specifically, lower extremity pain and back pain. In our adjusted models, neither the TPI nor lower extremity pain nor back pain explained the observed racial differences in gait velocity. In our group, African Americans had a higher rate of diabetes, which has been associated with decreased gait velocity. ${ }^{28}$ Again, adjustment for this comorbidity in our analyses did not explain our results.

Finally in our population, SES does not account for the differences seen in gait velocity. This is in agreement with prior studies of racial differences in various measures of physical functioning. In the Established Populations for Epidemiologic Studies of the Elderly cohort, differences in disability were attributable to SES in the North Carolina cohort but not the in New Haven cohort. ${ }^{8}$ Differences in disability rates in African Americans and Caucasians have been accounted for by education in some studies but not in others. ${ }^{4}{ }^{6}$ It is likely that SES contributes to some level of physical disability, where the more disadvantaged the population the higher the contribution. ${ }^{29}$ In our study we used education as a proxy for SES and found that overall, our population was fairly well educated. Even though there was a statistically significant difference in the years of education between African Americans and Caucasians, for the most part almost all participants completed at least 12 years of education 
or the equivalent of a high school diploma, thus the effect of education may have been blunted.

Our study, although highlighting the racial differences in gait velocity, has several important limitations. Over $50 \%$ of our original sample was excluded given incomplete data that would have precluded inclusion in multivariate analyses. Given the small sample size of African Americans and Caucasians, we do not have sufficient power to determine whether predictors of gait velocity vary significantly by race. Therefore this preliminary data is not generalizable to the greater US population. Also, while in this study we included many variables that are potentially related to gait and gait velocity, there may be other confounders that were not measured or included in this analysis that may in fact mediate the relationship between gait velocity and race. A comorbidity's presence or absence may not be sufficient to explain the differences in gait velocity. The decrease in gait velocity seen in African Americans may possibly be secondary to the severity or sequelae of certain comorbidities and not the presence of the condition itself. Unfortunately, the consequences of certain comorbidities, such as peripheral neuropathy in diabetes, were not measured in our study and cannot be included in our analyses.

Overall, we found that African Americans have decreased baseline gait velocity when compared to Caucasians in our sample of participants from the EAS. Although our sample size is small, limiting the generalizability of our data, these preliminary findings are very interesting given that elderly African Americans are at much higher risk of functional disability. Targeting gait velocity in this population may lead to improvements in functional decline. However, further studies are needed to confirm these results. Finding decreased baseline gait velocity in African Americans from another large cohort of elderly patients would corroborate our results. If this were in fact found to be true, clinical trials could be devised to determine if targeted interventions can indeed increase gait velocity, and if these increases lead to improved physical function. Although future studies are warranted, we feel that gait velocity is an important screening tool. Being aware of potential racial differences in this outcome may lead to more awareness of physical decline in certain at-risk populations, such as African Americans, and of the possible need for intervention.

\section{Acknowledgments}

Funding for Einstein Aging Study: NIH-NIA, AG03949

Funding for Irene Blanco: Mentored Clinical and Translational Research Career Development Award supported through an NIH Clinical and Translational Science Award (CTSA) grant, KL2RR025749, Albert Einstein College of Medicine

Sponsor's Role: None

\section{REFERENCES}

1. Sondik EJ, Huang DT, Klein RJ, et al. Progress toward the healthy people 2010 goals and objectives. Annu Rev Public Health. 2010; 31:271-281. 274 p folliwng 281. [PubMed: 20070194]

2. Arbeev KG, Butov AA, Manton KG, et al. Disability trends in gender and race groups of early retirement ages in the USA. Soz Praventivmed. 2004; 49:142-151. [PubMed: 15150866]

3. Bowen ME, Gonzalez HM. Racial/ethnic differences in the relationship between the use of health care services and functional disability: The health and retirement study (1992-2004). Gerontologist. 2008; 48:659-667. [PubMed: 18981282]

4. Thorpe RJ Jr, Weiss C, Xue QL, et al. Transitions among disability levels or death in African American and white older women. J Gerontol A Biol Sci Med Sci. 2009; 64:670-674. [PubMed: 19228790] 
5. Kelley-Moore JA, Ferraro KF. The black/white disability gap: Persistent inequality in later life? J Gerontol B Psychol Sci Soc Sci. 2004; 59:S34-S43. [PubMed: 14722342]

6. Liao Y, McGee DL, Cao G, et al. Black-white differences in disability and morbidity in the last years of life. Am. J. Epidemiol. 1999; 149:1097-1103. [PubMed: 10369503]

7. Mendes de Leon CF, Beckett LA, Fillenbaum GG, et al. Black-white differences in risk of becoming disabled and recovering from disability in old age: A longitudinal analysis of two EPESE populations. Am J Epidemiol. 1997; 145:488-497. [PubMed: 9063338]

8. Mendes de Leon CF, Fillenbaum GG, Williams CS, et al. Functional disability among elderly blacks and whites in two diverse areas: The New Haven and North Carolina EPESE. Established Populations for the Epidemiologic Studies of the Elderly. Am J Public Health. 1995; 85:994-998. [PubMed: 7604929]

9. Guralnik JM, Ferrucci L, Pieper CF, et al. Lower extremity function and subsequent disability: Consistency across studies, predictive models, and value of gait speed alone compared with the short physical performance battery. J Gerontol A Biol Sci Med Sci. 2000; 55:M221-M231. [PubMed: 10811152]

10. Rothman MD, Leo-Summers L, Gill TM. Prognostic significance of potential frailty criteria. J Am Geriatr Soc. 2008; 56:2211-2116. [PubMed: 19093920]

11. Studenski S, Perera S, Wallace D, et al. Physical performance measures in the clinical setting. J Am Geriatr Soc. 2003; 51:314-322. [PubMed: 12588574]

12. Montero-Odasso M, Schapira M, Soriano ER, et al. Gait velocity as a single predictor of adverse events in healthy seniors aged 75 years and older. J Gerontol A Biol Sci Med Sci. 2005; 60:13041309. [PubMed: 16282564]

13. Lipton RB, Katz MJ, Kuslansky G, et al. Screening for dementia by telephone using the memory impairment screen. J Am Geriatr Soc. 2003; 51:1382-1390. [PubMed: 14511157]

14. Verghese J, Katz MJ, Derby CA, et al. Reliability and validity of a telephone-based mobility assessment questionnaire. Age Ageing. 2004; 33:628-632. [PubMed: 15501841]

15. Yesavage JA, Brink TL, Rose TL, et al. Development and validation of a geriatric depression screening scale: A preliminary report. J Psychiatr Res. 1982; 17:37-49. [PubMed: 7183759]

16. Blessed G, Tomlinson BE, Roth $\mathrm{M}$. The association between quantitative measures of dementia and of senile change in the cerebral grey matter of elderly subjects. Br J Psychiatry. 1968; 114:797-811. [PubMed: 5662937]

17. Katona CL. Approaches to the management of depression in old age. Gerontology. 1994; 40(Suppl 1):5-9. [PubMed: 8020768]

18. Hall CB, Lipton RB, Sliwinski M, et al. Cognitive activities delay onset of memory decline in persons who develop dementia. Neurology. 2009; 73:356-361. [PubMed: 19652139]

19. Verghese J, Lipton RB, Hall CB, et al. Abnormality of gait as a predictor of non-Alzheimer's dementia. N Engl J Med. 2002; 347:1761-1768. [PubMed: 12456852]

20. Bilney B, Morris M, Webster K. Concurrent related validity of the GAITRite walkway system for quantification of the spatial and temporal parameters of gait. Gait Posture. 2003; 17:68-74. [PubMed: 12535728]

21. Cesari M, Kritchevsky SB, Penninx BW, et al. Prognostic value of usual gait speed in wellfunctioning older people--results from the Health, Aging and Body Composition Study. J Am Geriatr Soc. 2005; 53:1675-1680. [PubMed: 16181165]

22. Perera S, Mody SH, Woodman RC, et al. Meaningful change and responsiveness in common physical performance measures in older adults. J Am Geriatr Soc. 2006; 54:743-749. [PubMed: 16696738]

23. Studenski S, Perera S, Patel K, et al. Gait speed and survival in older adults. JAMA. 2011; 305:5058. [PubMed: 21205966]

24. Rolita L, Holtzer R, Wang C, et al. Homocysteine and mobility in older adults. J Am Geriatr Soc. 2010; 58:545-550. [PubMed: 20158558]

25. Burns R, Graney MJ, Lummus AC, et al. Differences of self-reported osteoarthritis disability and race. J Natl Med Assoc. 2007; 99:1046-1051. [PubMed: 17913116]

26. Sims EL, Keefe FJ, Kraus VB, et al. Racial differences in gait mechanics associated with knee osteoarthritis. Aging Clin Exp Res. 2009; 21:463-469. [PubMed: 20154517] 
27. Meghani SH, Cho E. Self-reported pain and utilization of pain treatment between minorities and nonminorities in the United States. Public Health Nurs. 2009; 26:307-316. [PubMed: 19573209]

28. Strotmeyer ES, de Rekeneire N, Schwartz AV, et al. The relationship of reduced peripheral nerve function and diabetes with physical performance in older white and black adults: The Health, Aging, and Body Composition (Health ABC) study. Diabetes Care. 2008; 31:1767-1772. [PubMed: 18535192]

29. Miller DK, Wolinsky FD, Malmstrom TK, et al. Inner city, middle-aged African Americans have excess frank and subclinical disability. J Gerontol A Biol Sci Med Sci. 2005; 60:207-212. [PubMed: 15814864] 
Table 1

Population Characteristics by Race

\begin{tabular}{|l|c|c|c|}
\hline & African Americans (n=56) & Caucasians (n=157) & p-value \\
\hline Age (median years, IQR) ${ }^{*}$ & $75.5(73.3-80.9)$ & $79.9(75.5-83.8)$ & $<0.01$ \\
\hline$\%$ Female $^{\dagger}$ & $80.4 \%$ & $59.9 \%$ & $<0.01$ \\
\hline Education (median years, IQR) & $12(11-15.5)$ & $14(12-18)$ & $<0.01$ \\
\hline BMI (mean \pm SD) ${ }^{*}$ & $28.9 \pm 6.4$ & $26.9 \pm 4.3$ & 0.03 \\
\hline Gait Velocity (cm/sec, mean \pm SD) & $90.19 \pm 17.87$ & $99.06 \pm 20.08$ & $<0.01$ \\
\hline Cognitive Impairment (\%) & $7.1 \%$ & $1.0 \%$ & 0.02 \\
\hline Depression (\%) & $10.7 \%$ & $8.3 \%$ & 0.59 \\
\hline Osteoarthritis (\%) & $73.2 \%$ & $77.1 \%$ & 0.59 \\
\hline COPD (\%) & $3.6 \%$ & $8.3 \%$ & 0.36 \\
\hline Diabetes (\%) & $28.6 \%$ & $13.4 \%$ & 0.01 \\
\hline Heart Attack (\%) & $3.6 \%$ & $7.6 \%$ & 0.37 \\
\hline Stroke (\%) & $8.9 \%$ & $8.3 \%$ & 1.0 \\
\hline Hip replacement or Pinning (\%) & $1.8 \%$ & $6.4 \%$ & 0.30 \\
\hline Hip/Femur/Pelvis Fracture (\%) & $0 \%$ & $2.6 \%$ & 0.58 \\
\hline Number of Comorbidities (median, IQR) & $1(1-2)$ & $1(1-2)$ & 0.83 \\
\hline Total Pain Index (median, IQR $)$ & $3.2(1.1-7.0)$ & $2.0(0.6-5.3)$ & 0.09 \\
\hline Back Pain (\%) & $26.8 \%$ & $35.7 \%$ & 0.25 \\
\hline Lower extremity pain (\%) & $37.5 \%$ & $36.3 \%$ & 0.87 \\
\hline
\end{tabular}

Non-normally distributed continuous variables were analyzed using the Mann-Whitney U test and reported as median (IQR).

Categorical variables were analyzed using the Chi-square test and reported as percentages.

${ }^{\dagger}$ Normally distributed continuous variables were analyzed using Student's t-test and reported as mean \pm SD 
Table 2

Bivariate Analyses Between Dichotomous Covariates and Gait Velocity*

\begin{tabular}{|c|c|c|c|}
\hline Variable & Yes & No & p-value \\
\hline Race: African American $\left(\right.$ mean \pm SD) ${ }^{\dagger}$ & $90.2 \pm 17.9$ & $99.1 \pm 20.1$ & $<0.01$ \\
\hline Gender: Male (mean $\pm \mathrm{SD})$ & $99.8 \pm 19.9$ & $95.1 \pm 19.7$ & 0.10 \\
\hline Memory Loss (median, IQR) ${ }^{*}$ & $107.4(81.5-109.4)$ & $97.7(82.7-109.8)$ & 0.72 \\
\hline Depression (median, IQR) & $89.3(77.3-101.1)$ & $98.5(82.8-110.1)$ & 0.04 \\
\hline Osteoarthritis (mean $\pm \mathrm{SD})$ & $97.8 \pm 19.6$ & $93.3 \pm 20.52$ & 0.15 \\
\hline COPD (median, IQR) & $97.0(88.1-107.0)$ & $97.8(82.0-109.8)$ & 0.92 \\
\hline Diabetes $($ mean $\pm \mathrm{SD})$ & $87.4 \pm 19.8$ & $98.7 \pm 19.4$ & $<0.01$ \\
\hline Heart Attack(median, IQR) & $96.5(83.5-120.1)$ & $98.0(82.0-109.7)$ & 0.97 \\
\hline Stroke (median, IQR) & $91.8(73.9-98.5)$ & $97.9(83.2-109.9)$ & 0.14 \\
\hline Hip replacement or Pinning (median, IQR) & $85.4(64.8-101.6)$ & $98.2(83.2-109.8)$ & 0.14 \\
\hline Hip/Femur/Pelvis Fracture (median, IQR) & $92.4(73.15-92.4)$ & $97.9(82.8-109.8)$ & 0.06 \\
\hline Back Pain $($ mean \pm SD) & $95.4 \pm 21.9$ & $97.3 \pm 18.8$ & 0.52 \\
\hline Lower extremity pain $($ mean \pm SD) & $92.1 \pm 19.2$ & $99.4 \pm 19.8$ & $<0.01$ \\
\hline
\end{tabular}

* All values listed above are for the mean or median gait velocity in $\mathrm{cm} / \mathrm{sec}$ with regards to the presence or absence of a specific variable.

${ }^{\dagger}$ Normally distributed continuous variables were analyzed using Student's t-test and reported as mean \pm SD

${ }^{*}$ Non-normally distributed continuous variables were analyzed using the Mann-Whitney U test and reported as median (IQR). 
Table 3

Linear Regression Models Explaining the Association of Race and Gait Velocity

\begin{tabular}{|l|c|c|}
\hline & $\beta$ for Race in cm/sec $^{*}$ & $\mathbf{p}$-Value for $\boldsymbol{\beta}$ \\
\hline Model 1: Unadjusted model with race as sole independent variable & -8.87 & $<0.01$ \\
\hline Model 2: Adjusted for race, age, gender, BMI & -8.49 & $<0.01$ \\
\hline Model 3: Adjusted for race, age, gender, BMI and education & -7.82 & 0.01 \\
\hline Model 4: Adjusted for race, age, gender, BMI, education and comorbidities ${ }^{\dagger}$ & -7.49 & 0.015 \\
\hline Model 5: Adjusted for race age, gender, BMI, education, comorbidities and pain scales ${ }^{\dagger}$ & -7.79 & 0.011 \\
\hline
\end{tabular}

* Caucasians are the reference group to which African Americans are compared to. A negative $\beta$ corresponds to a decrease in gait velocity.

Comorbidities included: Cognitive dysfunction, history of: osteoarthritis, diabetes, stroke, hip replacement or pinning, hip/pelvis/femur fracture

${ }^{*}$ Pain scales included: TPI, back pain, lower extremity pain 